\title{
Impact of Receiver Sensitivity on the Performance of Sensor Networks ${ }^{\dagger}$
}

\author{
Gianluigi Ferrari ${ }^{1,2}$, Ozan K. Tonguz ${ }^{1}$, and Megh Bhatt ${ }^{3}$ \\ ${ }^{1}$ Electrical and Computer Engineering Department, Carnegie Mellon University \\ Pittsburgh, PA, 15213-3890, USA - Phone: 412-268-5991 Fax: 412-268-2860 \\ ${ }^{2}$ Dipartimento di Ingegneria dell'Informazione, University of Parma \\ I-43100, Parma, Italy \\ ${ }^{3}$ CyberGuard Corporation, Fort Lauderdale, FL 33062, USA \\ E-mail: gferrari@cmu.edu, tonguz@ece.cmu.edu, mbhatt@cyberguard.com
}

\begin{abstract}
Medium access control (MAC) protocols significantly affect the performance of șensor networks. Little attention, however, has been devoted to the impact of physical layer characteristics on the MAC layer. One of the most important physical layer parameters is the receiver sensitivity. In this paper, we study the impact of receiver sensitivity on the network performance using a recently developed communication-theoretic framework for the analysis of multi-hop ad hoc wireless networks. In particular, we consider a reservation-based MAC protocol scheme, defined as reserve-listen-and-go (RESLIGO) without retransmission, suitable for circuit-switched sensor networks with limited receiver sensitivity. It will be shown that, in order to maximize the transfer of information in the network, the optimal receiver sensitivity depends on the traffic load.
\end{abstract}

I. INTRODUCTION

One of the important issues in sensor networks is the choice of the medium access scheme. Lack of central authority leads to interesting trade-offs and interactions between physical, medium access control (MAC), and routing layers of the network protocol stack. In particular, the impairments introduced by the wireless channel make the choice of the MAC protocol crucial. The use of multiple access schemes based on centralized division of the common radio resource (e.g., time division multiple access) is quite complex, due to synchronization reasons. Random access seems then a more appealing choice in ad hoc wireless networks. Random access MAC protocols for one-hop wireless networks, such as Aloha and carrier sense multiple access (CSMA), have been studied in great detail in the literature [1], [2]. The impact of unequal carrier sensing and transmitting ranges has been considered in [3], [4]. The effect of variations in network size, network density, and traffic load on the performance of an ad hoc wireless network using CSMA MAC protocol has also been studied [5].

In [6], [7], through a novel communication-theoretic approach to the analysis of ad hoc wireless networks, assuming a multi-hop circuit-switched network communication scenario with disjoint active multi-hop routes, two reservation-based

${ }^{\dagger} \mathrm{G}$. Ferrari and O. K. Tonguz were funded in part by Army Research Office (ARO) under Contract No. DAAD19-02-1-0389 and CYLAB of Camegie Mellon University (CMU). Any opinions, findings, and conclusions or recommendations expressed in this publication are those of the authors and do not necessarily reflect the views of the Army Research Office or CYLAB of $\mathrm{CMU}$.
MAC protocols, without retransmissions in intermediate links, have been proposed. The performance of these protocols has been evaluated in terms of bit error rate (BER) [7] and effective transport capacity [6]. In particular, the concept of effective transport capacity, introduced for the first time in [6], represents the bandwidth-distance product which is actually carried by the network-it obviously depends on the actual network traffic load. The basics of the two MAC protocols proposed in [6], [7] are outlined in the following.

- The first MAC protocol, defined as reserve-and-go (RESGO), is such that each node, after reserving a route, transmits without sensing the channel'. The receiver sensitivity is such that a node can hear only a neighboring node.

- The second MAC protocol, defined as reserve-listen-andgo (RESLIGO) is characterized by the fact that a node, after reserving a multi-hop route to its destination, senses the channel before transmitting: if no transmission is going on, then the node starts transmitting ${ }^{2}$. In this case, the receiver sensitivity at each node is assumed to be infinite.

In this paper, we extend RESLIGO MAC protocol, as proposed in [6], [7], to account for limited receiver sensitivity, which corresponds to a practical scenario. For the sake of notation simplicity, we still refer to this MAC protocol as RESLIGO (with limited receiver sensitivity), and we analyze the impact of its use on the performance of a circuit-switched sensor network in terms of BER and effective transport capacity. In particular, we show that, for large values of the receiver sensitivity, RESLIGO MAC protocol with limited receiver sensitivity reduces to RESGO MAC protocol, whereas for sufficiently low values of the receiver sensitivity it reduces to the original RESLIGO MAC protocol [6], [7]. For a given

\footnotetext{
${ }^{3}$ This MAC protocol was referred in [6], [7] as Aloha MAC protocol, for its resemblance, in terms of route activation independent from the activity of other nodes in the network. with the classical Aloha MAC protocol [1]. However, there are significant differences which make the proposed protocol different from the classical Aloha MAC protocol: (i) multi-hop route reservation and (ii) no use of retransmission techniques.

${ }^{2}$ This MAC protocol was referred in [6], [7] as per-route carrier sense multiple access (PR-CSMA) MAC protocol, for its resemblance, in terms of route activation after sensing. with the classic CSMA MAC protocol [2].
} 
value of the traffic load, there exist optimal values of transmission data-rate and receiver sensitivity for the maximization of the effective transport capacity. Hence, this result provides valuable directions for network optimization.

\section{Preliminaries: Circuit Switched Sensor NETWORKS}

\section{A. Basic Assumptions}

The considered network communication model is based on the following assumptions.

- No retransmission mechanism is considered at intermediate relay nodes.

- Circuit switching with multiple disjoint routes is considered. In particular, a node can act as a relay node in a single route at a time.

- No buffering is considered, and the generation process coincides with the transmission process. For example, after reserving a route to a desired destination a sensor node measures a physical quantity of interest and transmits immediately the corresponding data.

- The transmission is packetized, and the packets have fixed dimension ( $L$ bits per packet).

- The transmission data-rate $R_{b}$ (dimension: $[\mathrm{b} / \mathrm{s}]$ ) is fixed.

- The generation/transmission process at each node, after route reservation, is Poisson distributed. We indicate the average packet generation rate as $\lambda$ (dimension: [pck/s]) A necessary condition that needs to be satisfied is that the average inter-arrival time between two consecutive packet generations must be shorter than the packet duration, i.e. $1 / \lambda<L / R_{b}$.

- The route creation phase is not considered in detail, since it is beyond the scope of this paper. However, we point out that it should be based on broadcast percolation [8][10].

- The nodes are static and lay at the vertices of a perfectly uniform grid structure. The node regularity allows to isolate and evaluate the sole impact of receiver sensitivity. Moreover, this model may be an accurate model for particular fixed sensor networks.

\section{B. Communication-Theoretic Principles}

We assume that $N$ nodes are placed at the vertices of a square grid inside a circular ${ }^{3}$ area $A$. Defining by $\rho_{S} \triangleq N / A$ the node spatial density, the minimum inter-node distance can be written as $r_{L} \approx 1 / \sqrt{\rho_{S}}$ [6], [7]. Denoting by $\mathrm{BER}_{L}$ the $B E R$ at the end of a minimum length link, it is possible to show that the BER at the end of the $n$-th link of a multi-hop route can be written as

$$
\mathrm{BER}^{(n)} \approx 1-\left(1-\mathrm{BER}_{L}\right)^{n} .
$$

An expression for the average BER can be obtained by evaluating (1) for an average number of hops $\bar{n}_{h}$. Assuming

\footnotetext{
${ }^{3}$ For sufficiently large number of nodes $N$, as will be considered in the remainder of the paper, the shape of the network area has very little influence on the performance. In other words, the obtained results hold, but with minor quantitative changes, for any shape of the network area.
}

that the number of hops is uniformly distributed between one and the maximum number over a diameter of the circular network area, it is possible to show that $\bar{n}_{h}=\lfloor\sqrt{N / \pi}\rceil$, where the notation $[*\rceil$ indicates the integer value closest to $*[6]$, [7]. Denoting by $\overline{\mathrm{BER}}$ the average BER, it follows that

$$
\overline{\mathrm{BER}}=\mathrm{BER}^{\left(\bar{n}_{h}\right)} \approx 1-\left(1-\mathrm{BER}_{L}\right)^{|\sqrt{N / \pi}|} .
$$

Note that the link BER depends on the signal-to-noise ratio (SNR) at the ending node of the link and on the characteristics of the transmission channel. We assume that the transmitted signal is affected by free-space loss. Hence, according to Friis formula [11], the received signal power at distance $d$ from the transmitter, denoted by $P_{r}^{(d)}$, has the following expression:

$$
P_{r}^{(d)}=\frac{\alpha P_{t}}{d^{2}}=\frac{G_{t} G_{r} c^{2} P_{t}}{(4 \pi)^{2} f_{l} f_{c}^{2} d^{2}}
$$

where: $P_{t}$ (dimension: [W]) is the transmitted power from each node; $G_{t}$ and $G_{r}$ are the transmitter and receiver antenna gains; $f_{c}$ (dimènsion: [Hz]) is the carrier frequency; $c \approx 3 \times$ $10^{8} \mathrm{~m} / \mathrm{s}$ is the speed of light; and $f_{l} \geq 1$ is a loss factor.

In the remainder of this paper, we will consider uncoded binary phase shift keying (BPSK) signaling ${ }^{4}$, in which case $\mathrm{BER}_{L}=Q\left(\sqrt{2 \mathrm{SNR}_{L}}\right)=(1 / \sqrt{2 \pi}) \int_{\sqrt{2 \mathrm{SNR}_{L}}}^{\infty} \exp \left(-x^{2} / 2\right) d x$ where $\mathrm{SNR}_{L}$ is the link $\mathrm{SNR}$. In a realistic radio network communication scenario, the link SNR can be written as

$$
\mathrm{SNR}_{L}=\frac{P_{r}^{\left(r_{L}\right)}}{P_{\text {thermal }}+P_{I N T}}
$$

where $P_{\text {thermal }}$ is the thermal noise power and $P_{Y N T}$ represents the interference power. In particular, the thermal noise power can be expressed as $P_{\text {thermal }}=F k T_{0} B$, where $F$ is the noise figure [11], $k=1.38 \times 10^{-23} \mathrm{~J} / \mathrm{K}$ is the Boltzmann's constant, $T_{0}$ is the room temperature $\left(T_{0} \approx 300 \mathrm{~K}\right)$, and $B$ is the transmission bandwidth. In the case of uncoded BPSK signaling, $B \approx R_{b}$. The interference power $P_{I N T}$ depends, in addition to the node geometry, on the MAC protocol [6], [7]. The performance in an ideal network communication scenario is obtained by setting $P_{I N T}=0$. In order to make a simple analysis, it is expedient to use the same value of $P_{I N T}$ for all links of a multi-hop route. This is obviously incorrect, since, depending on their positions in the sensor network, different links could experience different interference levels. Hence, we consider a worst-case interference analysis, by evaluating the interference power at the ending node of a link placed in the center of the network.

\section{RESLIGO MAC PROTOCOL WITH LIMITED RECEIVER SENSITIVITY}

A network communication scenario can be characterized in terms of receiver sensitivity, denoted by $S_{\min }$ and defined as the minimum received power necessary for a received signal to be distinguished from background noise. The lower the receiver sensitivity, the more sensitive is a receiver.

\footnotetext{
${ }^{4}$ Extensions of the proposed work to the case of different modulation formats are straightforward, by suitably changing the expression of the link BER
} 


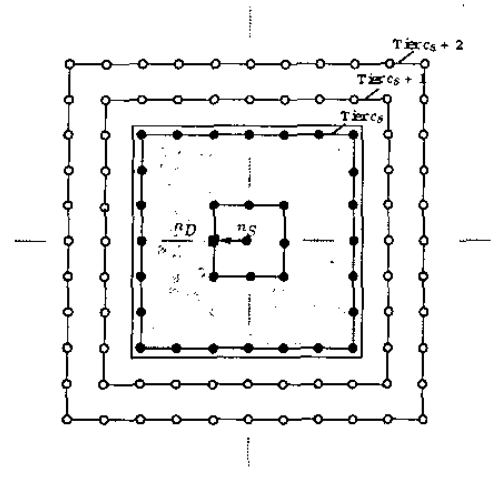

Fig. 1. Carrier sensing region (shaded region) relative to node $n_{S}$.

A. Carrier Sensing Range and Carrier Sensing Area

Given the receiver sensitivity of a node in the network, it is possible to determine the carrier sensing range, defined as the distance at which the transmitted power reduces, because of free space propagation loss, to $S_{\min }$. Indicating by $r_{c s}$ the carrier sensing range, using Friis formula it follows that

$$
r_{c s}=\sqrt{\frac{\alpha P_{t}}{S_{\min }}}
$$

Obviously, the received power between two neighboring nodes has to be larger than the receiver sensitivity, i.e., the following condition must be satisfied for the network to properly work:

$$
\alpha P_{t} \rho_{S} \geq S_{\min }
$$

We refer to the area, surrounding a node, inside which nodes can "hear" the central node, as carrier sensing area. For ease of analysis, we assume that the carrier sensing area is squarequalitatively, the obtained results hold also in the case of circular carrier sensing area, which corresponds to the case of nodes equipped with omnidirectional antennas. In Fig. 1, the carrier sensing area relative to node $n_{S}$ is indicated as a shaded area. As one can immediately see, the carrier sensing area includes a finite number of concentric tiers, from order 1 to order $c_{s}$, where

$$
c_{s} \triangleq\left\lfloor\frac{r_{c s}}{r_{L}}\right\rfloor \text {. }
$$

\section{B. Interference Power}

Based on the notions of carrier sensing range and carrier sensing area, it is possible to derive an expression for the interference power experienced at the ending node of a link in the center of the network (for instance, the link between $n_{S}$ and $n_{D}$ in Fig. 1). It is immediate to conclude that the vulnerable time interval for a given node in the network will depend on its current position, and can be obtained by extending to this case the analysis conducted in the case of RESLIGO MAC protocol. It is possible to show that in this case the interference power can be written as

$$
\begin{aligned}
P_{I N T}^{R E S L I G O} \approx & \alpha P_{t} \rho_{S}\left[\Delta_{C f}^{\prime}\left(N, \rho_{S}, R_{b}, S_{\min }\right)\right. \\
& \left.+\Delta_{A f}\left(N, S_{\min }\right)\right]
\end{aligned}
$$

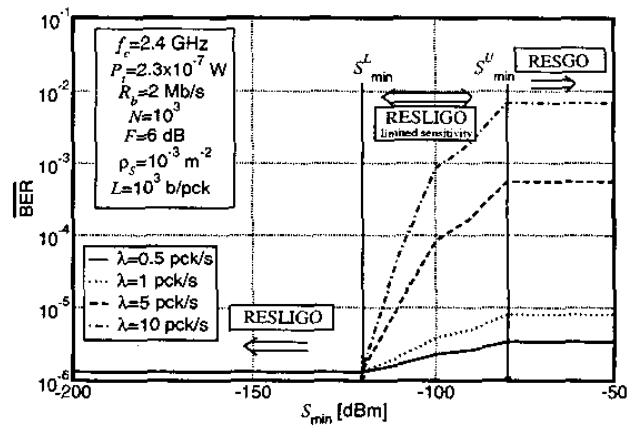

Fig. 2. BER performance versus receiver sensitivity. Various values of the average packet generation rate $\lambda$ are considered.

where

$$
\Delta_{A f}\left(N, S_{\min }\right) \triangleq \sum_{i=c_{s}+1}^{i_{\max }}\left(\frac{6}{i^{2}}+8 \sum_{j=1}^{i-1} \frac{1}{i^{2}+j^{2}}\right)-1
$$

and

$$
\begin{aligned}
& \Delta_{C f}^{\prime}\left(N, \rho_{S}, R_{b}, S_{\min }\right) \triangleq \sum_{i=1}^{c_{s}}\left[\frac{4}{i^{2}}\left(1-e^{-\lambda 2 i \tau_{m}}\right)\right. \\
&\left.+\frac{2}{i^{2}}\left(1-e^{-\lambda 2 \sqrt{2} i \tau_{m}}\right)+8 \sum_{s=1}^{i-1} \frac{1}{i^{2}+s^{2}}\left(1-e^{-\lambda 2 \sqrt{i^{2}+s^{2}} \tau_{m}}\right)\right] \\
&-\left(1-e^{-\lambda 2 \tau_{m}}\right)
\end{aligned}
$$

where $i_{\max }=\sqrt{N} / 2$ and $\tau_{m}=r_{L} / c$ is the propagation time between two neighboring nodes.

\section{BIT ERror Rate PERFORMANCE}

We evaluate the BER as a function of the receiver sensitivity, considering a fixed node spatial density $\left(\rho_{S}=2 \times 10^{-3}\right.$ $\mathrm{m}^{-2}$ ) and a fixed number of nodes ( $N=10^{3}$ nodes). The obtained results are shown in Fig. 2, where various values of the packet generation rate $\lambda$ are considered. The obtained BER curves have a characteristic behavior, which allows to clearly distinguish three regions

- There exists a critical minimum receiver sensitivity value, indicated as $S_{\min }^{L}$, below which the BER is the same, regardless of the packet generation rate. In this case, the receiver sensitivity is such that only a single route at a time is active in the network, i.e., this corresponds to the case of RESLIGO MAC protocol as proposed in [6], [7]. For the considered values of the major network parameters in Fig. 2, $S_{\min }^{L} \approx-120 \mathrm{dBm}$. From a practical point of view, the condition $S_{\min }=-\infty$ corresponds to the condition $S_{\min }<S_{\min }^{L}$.

- There exists another critical value of the receiver sensitivity, indicated as $S_{\min }^{U}$, above which the BER is constant with respect to $S_{\min }$, and depends only on the packet generation rate. In this case, a node can only hear its immediate neighbors. This scenario corresponds to the case of a sensor network where RESGO MAC protocol is being used. For the considered values of the major network parameters in Fig. 2, $S_{\min }^{U} \approx-80 \mathrm{dBm}$. 
- Finally, there is an intermediate region, for $S_{\min }^{L}<$ $S_{\min }<S_{\min }^{U}$, where the BER performance depends on both packet generation rate and receiver sensitivity.

From the results in Fig. 2, it is immediate to conclude that, for a given value of the node spatial density $\rho_{S}$, there exists a minimum possible achievable BER. In particular, there exists a minimum critical receiver sensitivity $S_{\min }^{L}$ such that the minimum possible BER can be obtained by using a receiver sensitivity lower than $S_{\min }^{L}$.

\section{EFFECTIVE TRANSPORT CAPACITY}

Transport capacity has recently been proposed as a metric for evaluating the performance of ad hoc wireless networks [12], since it represents a measure of the traffic hauling capacity of the network. In [6], the concept of effective transport capacity, defined as the actual bandwidth-distance product carried by the network, is introduced. In the following, we extend the analysis proposed in [6] to evaluate the effective transport capacity (and, consequently, its overall maximum corresponding to the transport capacity) in a network communication scenario with RESLIGO MAC protocol.

\section{A. Derivation}

Based on the approach proposed in [6], the effective transport capacity in a circuit-switched ad hoc wireless network, where simultaneously active multi-hop routes are disjoint, can be generally written as follows:

$$
C_{T, e} \triangleq \lambda L N_{a r} \bar{n}_{s h} r_{L}
$$

where $N_{a r}$ represents the number of disjoint multi-hop routes simultaneously active and $\bar{n}_{s h}$ is the average sustainable number of hops. The idea behind the derivation of (11) is simple and intuitive. Since the multi-hop routes are disjoint, in each one of them the effective bit information flow is generated at the source node of the route (at average rate $\lambda L$ ). The average sustainable number of hops is defined as follows [6], [7]:

$$
\bar{n}_{s h} \triangleq \min \left\{\bar{n}_{h}, n_{s h}^{\max }\right\}
$$

where $\bar{n}_{h}$ is the average number of hops (an expression for which has been given before) and $n_{s h}^{\max }$ represents the maximum sustainable number of hops according to the maximum prescribed final BER. More precisely, indicating by $\mathrm{BER}^{\max }$ the maximum acceptable BER, from (1) it is immediate to obtain that

$$
n_{s h}^{\max }=\left\lfloor\frac{\ln \left(1-\mathrm{BER}^{\max }\right)}{\ln \left(1-\mathrm{BER}_{L}\right)}\right\rfloor .
$$

The condition on the maximum acceptable BER at the end of a multi-hop route (embedded into the average sustainable number of hops) can be interpreted as a condition on the quality of service (QOS) which is guaranteed, on average, by the network. Hence, the product $\lambda L \bar{n}_{s h} r_{L}$ represents the average effective transport capacity (bandwidth-distance product) associated with a single multi-hop route under a given QoS constraint. The total aggregate effective transport

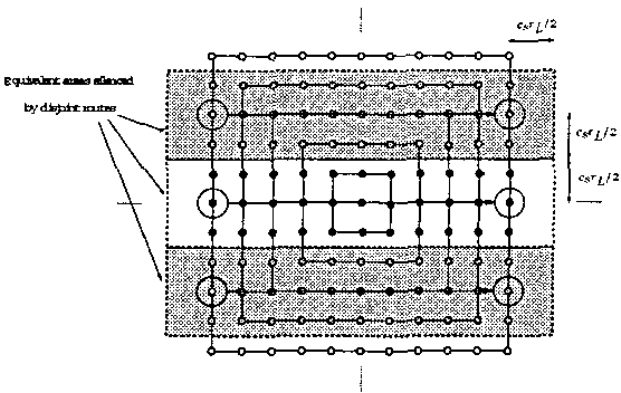

Fig. 3. Overlapping regions silenced by disjoint active multi-hop routes.

capacity supported by the network is then obtained by simply adding the contributions of the $N_{a r}$ active multi-hop routesin particular, we will show later that the number of active routes depends on the receiver sensitivity.

We now analyze the number $N_{a r}$ of simultaneously active multi-hop communication routes. In particular, based on the definition of receiver sensitivity and carrier sensing area, it is possible to determine the effective area "silenced" by a single active route, and, consequently, to determine how many routes can be be simultaneously active. Assuming straight multi-hop routes, as shown in in Fig. 3, it is possible to show that the maximum average number of simultaneously active routes is

$$
N_{a r}^{\text {RESLIGO }} \approx \frac{N}{c_{s}\left(\bar{n}_{h}+c_{s}\right)} .
$$

For decreasing values of the receiver sensitivity, $c_{s}$ increases, and the RESLIGO MAC protocol with limited receiver sensitivity reduces to the RESLIGO MAC protocol in [6], [7] when $N_{a r}^{\mathrm{RESLIGO}} \approx 1$, i.e., when $N \approx c_{s}\left(\overline{n_{h}}+c_{s}\right)$.

\section{B. Numerical Results}

In Fig. 4, the maximum effective transport capacity obtainable for each value of the average packet generation rate is shown. Various values of the receiver sensitivity are considered, and the performance in the ideal (no INI) case is also shown. As one can see from the figure, for decreasing values of the receiver sensitivity, the corresponding maximum effective transport capacity curve shifts from left to right. In particular, for sufficiently high values of the receiver sensitivity ( $S_{\min } \geq-80 \mathrm{dBm}$ with the numerical values used in the figure for the network parameters), the performance with RESLIGO MAC protocol coincides with that obtained using RESGO MAC protocol, whereas for sufficiently low values of the receiver sensitivity $\left(S_{\min } \leq-140 \mathrm{dBm}\right)$, the RESLIGO MAC protocol with limited receiver sensitivity reduces to the RESLIGO MAC protocol as proposed in [6], [7]. Hence, for small traffic loads, it is beneficial to use a relatively high receiver sensitivity, since the corresponding allowed spatial reuse significantly increases the effective transport capacity. On the other hand, for large traffic loads, the effective transport capacity is maximized by sufficiently reducing the receiver sensitivity, i.e., by reducing the number of simultaneously active routes, and, correspondingly, the inter-node interference. 


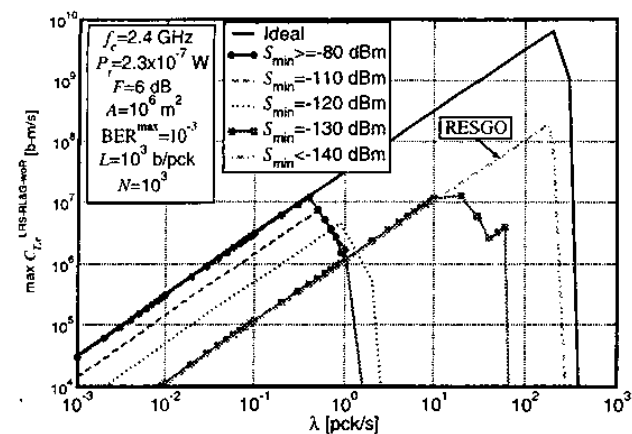

Fig. 4. Maximum effective transport capacity versus average packet generation rate, in the case with RESLIGO MAC protocol. Various values of the receiver sensitivity are considered. For comparison, the maximum effective transport capacity in the ideal (no INI) case is also shown.

In Fig. 5, the effective transport capacity is shown as a function of the number of nodes in the network, for fixed average packet generation rate $(\lambda=1 \mathrm{pck} / \mathrm{s})$ and receiver sensitivity $\left(S_{\min }=-120 \mathrm{dBm}\right)$, and for various values of the transmitted power. In all considered cases, at some point the effective transport capacity curve becomes constant (and highest): this corresponds to the case of fully connected sensor networks, i.e., networks where $\bar{n}_{s h}=\bar{n}_{h}$. According to the results in the figure, for large number of nodes, the larger effective transport capacity is obtained by reducing the transmitted power. This counter-intuitive result can be explained as follows. We first note that the network area is constant, so that for increasing value of the number of nodes, the node spatial density increases proportionally. Assuming that the considered receiver sensitivity allows a significant spatial reuse (the values of the network parameters considered in Fig. 5 satisfy this condition), it is reasonable to assume that $\bar{n}_{h} \gg c_{s}$, so that

$$
N_{a r}^{\mathrm{RESLIGO}} \approx \frac{N}{c_{s} \bar{n}_{h}} .
$$

Since we are considering the case of a fully connected network, recalling the definition of $c_{s}$ in (7), the effective transport capacity (11) can be written as

$$
C_{T, e}^{\mathrm{RESLIGO}} \approx \lambda L A \sqrt{\frac{S_{\min }}{\alpha P_{t}}} .
$$

Hence, for fixed values of $A, \lambda$, and $S_{\min }$, the effective transport capacity for large $N$ is inversely proportional to $\sqrt{P_{t}}$.

\section{CONCLUDING REMARKS}

In this paper, we have evaluated the impact of receiver sensitivity on the performance of circuit switched sensor networks. The MAC protocol considered, referred to as RESLIGO, and the corresponding network performance has been analyzed in terms of (i) BER at the end of an average multi-hop route and (ii) effective transport capacity. It has been shown that considering more and more sensitive receivers does not necessarily improve the network performance. In particular, in order to maximize the effective transport capacity, the optimal

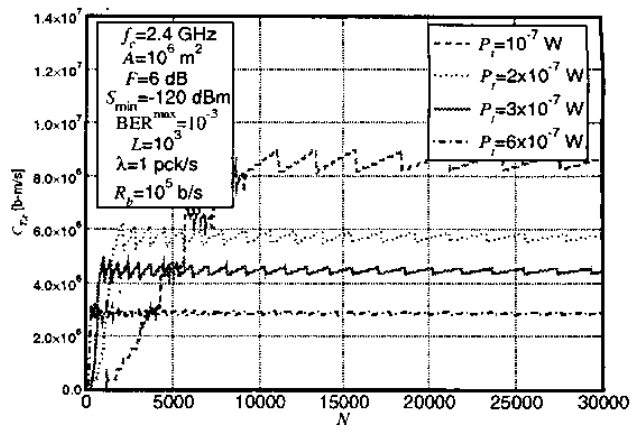

Fig. 5. Effective transport capacity versus number of nodes. for various values of the transmitted power. Note that the network area is fixed.

receiver sensitivity depends on the values of the traffic load and transmission data-rate:

- the highest possible effective transport capacity is obtained by considering very sensitive receivers: in this case, only one route at a time is active in the network and the traffic load can be increased significantly;

- on the other hand, for small traffic loads, the use of less sensitive receivers entails no degradation, in terms the effective transport capacity, with respect to the ideal (no INI) case (by allowing the spatial reuse).

For connected sensor networks with a large number of nodes, it has been shown that, reducing the transmitted power might have a beneficial effect in terms of effective transport capacity. This seems to be a counter-intuitive yet important result.

\section{REFERENCES}

[1] N. Abramson, "The throughput of packet broadcasting channels," IEEE Trans. Commun., vol. 25, pp. 117-128, January 1977.

[2] L. Kleinrock and F. A. Tobagi, "Packet switching in radio channels: Part I-Carrier sense multiple-access modes and their throughput-delay characteristics," IEEE Trans. Commun., vol. 23, no. 12, pp. 1400-1416, December 1975 .

[3] E. Jung and N. Vaidya, "A power control MAC protocol for ad hoc networks," in Proc. ACM Intern. Conf. on Mobile Comput. and Networking (MOBICOM), September 2002.

[4] J. Li, C. Blake, D. S. J. D. Couto, H. I. Lee, and R. Morris, "Capacity of ad hoc wireless networks." in Proc. ACM Intem. Conf. on Mobile Comput. and Networking (MOBICOM), Rome, Italy, July 2001, pp. 6169.

[5] R. Hekmat and P. V. Mieghem. "Interference in wireless multi-hop adhoc networks," in $\mathrm{Med}$-Hoc-Net, Sardegna. Italy, September 2002.

[6] G. Ferrari and O. K. Tonguz, "MAC protocols and transport capacity in ad hoc wireless networks: Aloha versus PR-CSMA," in Proc. IEEE Military Comm. Conf. (MILCOM), Boston, USA, October 2003.

[7] — "Performance of circuit-switched ad hoc wireless networks with Aloha and PR-CSMA MAC protocols," in Proc. IEEE Global Telecom. mun. Conf. (GLOBECOM), San Francisco, USA, December 2003.

[8] Y.-C. Cheng and T. G. Robertazzi, "Critical connectivity phenomena in multihop radio models," IEEE Trans. Commun., vol. 37, no. 7, pp. 770-777, July 1989.

[9] M. Sahimi, Applications of Percolation Theory. London, UK: Taylor\&Francis, 1994.

[10] R. Meester and R. Roy, Continuum Percolation. Cambridge, U.K.: Cambridge University Press, 1996.

[11] T. S. Rappaport, Wireless Communications. Principles \& Practice. Upper Saddle River, NJ, U.S.A.: Prentice-Hall, 2002, second edition.

[12] P. Gupta and P. R. Kumar, "The capacity of wireless networks," IEEE Trans. Inform. Theory, vol. 46, pp. 388-404, March 2000. 